\title{
Vibration mitigation of railway bridges using adaptive damping control
}

\author{
Andreas ANDERSSON \\ Ph.D., Researcher \\ Royal Institute of \\ Technology (KTH), \\ Stockholm, Sweden \\ adde@kth.se
}

\author{
Raid KAROUMI \\ Professor \\ Royal Institute of \\ Technology (KTH), \\ Stockholm, Sweden \\ raidk@kth.se
}

\author{
Alan O'CONNOR \\ Associate Professor \\ Trinity College Dublin, \\ Roughan \& O'Donovan \\ Innovative Solutions \\ Dublin, Ireland \\ oconnoaj@tcd.ie
}

\section{Summary}

In this paper, the advantage of an adaptive damping system is presented. A damper with variable stiffness is tuned based on estimates of the real-time frequency response, facilitating optimal vibration mitigation. The performance of the developed routines is investigated on an existing tied arch railway bridge. Based on previous field measurements, resonant behaviour of several hangers was found. In combination with low structural damping, the induced stresses resulted in a reduced fatigue service life. Passive dampers are currently installed on the longer hangers, each tuned to the fundamental natural frequency of the individual hanger. However, increased axial force during train passage results in a significant variation in natural frequency, with an apparent risk of detuning the passive dampers. The predicted performance of an adaptive damping system to account for this variation in dynamic behaviour is presented and its potential application is discussed.

Keywords: Railway bridge dynamics; tuned mass damper; adaptive control; finite element method.

\section{Introduction}

There is a constant demand on the railway authorities to increase both the allowable axle loads and the allowable speed on existing railway lines. An increased utilization factor of the bridges can often be justified based on refined capacity assessment and field measurements. For bridges susceptible to high dynamic amplification or resonance, a moderate increase in speed or axle load may result in a significant increase in utilization. For cases of excessive vibrations, external damping systems may be called for. Such systems often have a rather narrow bandwidth, making them sensitive to any mismatch in tuning frequency. This is especially pronounced for cases of low structural damping, which is often the reason for installing damper systems in the first place. Even a small change in either structural frequency or loading frequency may result in a significant disimprovement in vibration mitigation. In some cases a detuned damper may even result in a magnified dynamic response compared to the case of no external damping system. The bandwidth may be widened either by increasing the damping ratio or by installing multiple dampers with different tuning frequencies. Another, often more efficient alternative, is to use a damper with variable properties that via a control system analyses the real-time response to retune the damper.

\section{Tuned mass damper systems}

One of the most established methods for external damping systems is the tuned mass damper (TMD). The concept is to add a suspended mass to an existing structure and tune the natural frequency of the suspended mass to the dominant frequency of the main structure. If the vibration of the suspended mass is out of phase compared to the main structure, a counteracting inertia force will attenuate the vibration of the resulting system. The theory of the TMD was first established by [7] and much research has been devoted to find configurations for optimal vibration mitigation. The most decisive parameters are the mass ratio $\gamma=m_{\mathrm{TMD}} / m_{\text {structure }}$ and the damping ratio of the TMD. A 
mass ratio of a few percent often results in sufficient vibration mitigation. For global damping of large structures, e.g. bridges or buildings, this may still result in rather large dampers.

\subsection{SDOF and MDOF models}

Structures dominated by a single frequency may be simplified to a single degree of freedom (SDOF) system, Fig. 1a. The natural frequency and viscous damping of the SDOF-system is given by Eq. (1). For the case of a simply supported beam, Eq. (2), the equivalent stiffness $k_{1}$ and modal mass $m_{1}$ are governed by Eq. (3). Including the TMD results in a 2DOF-system illustrated in Fig. 1b and the equation of motion can be expressed according to Eq. (4). This simple system is suitable for parametric studies and for finding optimal damping parameters. The parameters are often evaluated based on the steady-state response. For the 2DOF-model in Fig. 1b, a closed solution can be found in [9]. For more complicated systems the equilibrium equation for each circular frequency $\omega$ can be solved using Eq. (5), implemented in many commercial finite element packages.

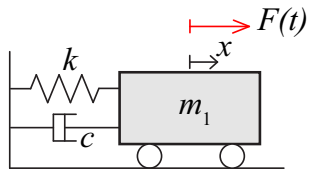

a)

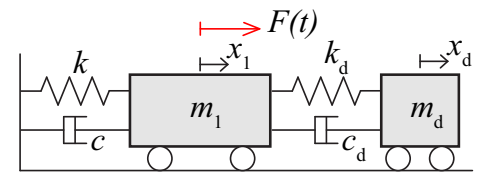

b)

Fig. 1: a) SDOF-model, b) 2DOF model of the same system with a TMD.

$$
\begin{aligned}
& f_{\text {SDOF }}=\frac{1}{2 \pi} \sqrt{\frac{k}{m_{1}}}, c=2 \zeta \sqrt{m_{1} k} \\
& f_{\text {beam }}=\frac{\pi}{2} \sqrt{\frac{E I}{m_{\text {beam }} L^{4}}}, \delta_{\text {beam }}=\frac{q L^{3}}{48 E I}, q=m_{\text {beam }} g L \\
& q=k \cdot \delta \rightarrow k=\frac{48 E I}{L^{3}}, f_{\text {SDOF }}=f_{\text {beam }} \rightarrow m_{1}=\frac{48 m_{\text {beam }} L}{\pi^{4}} \\
& {\left[\begin{array}{cc}
m_{1} & 0 \\
0 & m_{\mathrm{d}}
\end{array}\right]\left\{\begin{array}{l}
\ddot{x}_{1} \\
\ddot{x}_{\mathrm{d}}
\end{array}\right\}+\left[\begin{array}{cc}
c+c_{\mathrm{d}} & -c_{\mathrm{d}} \\
-c_{\mathrm{d}} & c_{\mathrm{d}}
\end{array}\right]\left\{\begin{array}{l}
\dot{x}_{1} \\
\dot{x}_{\mathrm{d}}
\end{array}\right\}+\left[\begin{array}{cc}
k+k_{\mathrm{d}} & -k_{\mathrm{d}} \\
-k_{\mathrm{d}} & k_{\mathrm{d}}
\end{array}\right]\left\{\begin{array}{l}
x_{1} \\
x_{\mathrm{d}}
\end{array}\right\}=\left\{\begin{array}{c}
F(t) \\
0
\end{array}\right\}} \\
& \left(\begin{array}{ll}
\left.-\omega^{2} \mathbf{M}+i \omega \mathbf{C}+\mathbf{K}\right) \mathbf{x}=\mathbf{F}
\end{array}\right.
\end{aligned}
$$

A precursor of the TMD is the pendulum damper, depicted in Fig. 2. It is often based on the same concepts as the TMD, i.e. to create a vibrating mass phase-shifted to the primary system. The natural frequency of the classical pinned pendulum is given by Eq. (6). If the mass of the rod is negligible compared to the mass of the bob, the frequency depends solely on the length of the pendulum. For the clamped pendulum, the natural frequency is obtained by considering a cantilever beam with a lumped end mass, resulting in Eq. (7). Damping can be added to the pendulum based on an SDOF approach, Eq. (8). It shall be noted that for the MDOF-system in Fig. 2b, the motion of the pendulum depends on the motion of the primary system. Not accounting for this may cause the damper to be partly detuned. In numerical simulations of pendulum motions, a nonlinear analysis is normally required. 


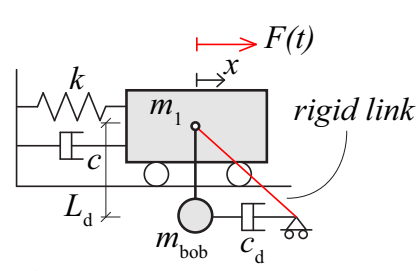

a)

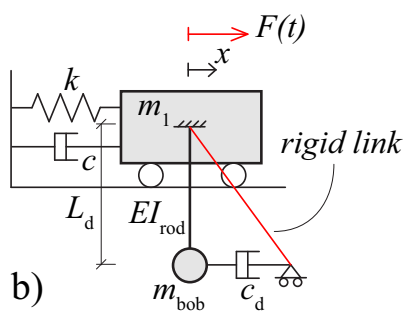

b)

Fig. 2: Pendulum damper models, a) pinned pendulum, b) clamped pendulum.

$$
\begin{aligned}
& f_{\mathrm{p}, \text { pinned }}=\frac{1}{2 \pi} \sqrt{\frac{g}{L_{\mathrm{d}}} \sqrt{\frac{m_{\mathrm{bob}}}{m_{\mathrm{bob}}+m_{\mathrm{rod}} / 3}}} \\
& f_{\mathrm{p}, \text { clamped }}=\frac{1}{2 \pi} \sqrt{\frac{3 E I_{\mathrm{rod}}}{m_{\mathrm{bob}} L_{d}{ }^{3}+\frac{48 m_{\mathrm{rod}} L_{d}^{3}}{\pi^{4}}\left(3-\frac{8}{\pi}\right)}} \\
& c_{\mathrm{d}}=2 \zeta \sqrt{m_{\mathrm{d}} k_{\mathrm{d}}}, \quad k_{\mathrm{d}}=m_{\mathrm{d}} \omega_{\mathrm{d}}{ }^{2}, \quad m_{\mathrm{d}}=m_{\mathrm{bob}}
\end{aligned}
$$

\subsection{Adaptive and semi-active control}

Vibration control systems are often divided in passive systems, adaptive/semi-active control and active control. A passive system is not able to change properties and is most suitable for timeinvariant loading. An adaptive control system can change properties of the damper but with a significantly narrower bandwidth than the vibration system, i.e. a relatively slow change in properties compared to the dynamics of the structure. A semi-active system however has a significantly higher bandwidth and can change properties several times during a single period of vibration, [4]. In a fully active system, a force control is obtained by a load actuator. One of the benefits of the adaptive and semi-active systems is that they need only a fraction of the energy input compared to a fully active system.

Some adaptive systems are based on stiffness control, with the objective to retune the frequency of the TMD for optimal vibration mitigation. In practical applications, the variable stiffness component often consists of non-trivial mechanical devices with multiple moving parts and a rather limited frequency range. In comparison, semi-active systems are often based on damping control. This can be accomplished by magnetorheological devices, which are able to rapidly change properties due to a small controllable electrical current. Semi-active and active control systems originate from the use of shock absorbers but can also be implemented for TMD applications. The principle of controllable shock absorbers is to create a force that at any increment of time counteracts the motion of the structure. In comparison, a TMD produces a force based on its natural frequency, which counteracts the corresponding frequency of the structure due to its phase shift.

Variable stiffness control is based on incremental update of the present dominant frequencies. The two most common time-frequency estimators are the Short Time Fourier Transform (STFT) and the Short Time Wavelet Transform (STWT). Both operate on a short time signal prior to present time and the performance of the system depends on how rapidly and accurately the frequency content can be estimated. The frequency resolution of the STFT method can be increased by zero-padding or copying the signal, without adding additional information in the signal. Using wavelets, arbitrary frequency resolution can be obtained by scaling. However, the accuracy in both methods is still limited to the information contained in the studied signal. The final performance is therefore a trade-off between response time and frequency resolution. 


\section{Case study bridge}

The use of passive and adaptive damping systems are studied on an existing railway bridge by means of numerical simulations. The bridge has earlier been the subject of dynamic assessments and its dynamic behaviour has been verified by field measurements [1,5]. The objective of this paper is to illustrate the potential of an adaptive damping system for vibration mitigation.

\subsection{Bridge properties}

The bridge is located approximately $110 \mathrm{~km}$ West of Sundsvall, a municipality in central Sweden. It is part of the Northern main line and is important for ore transports as well as commuter trains. It was built in 1959 and is designed as a single span single track tied arch railway bridge. The span is $45 \mathrm{~m}$. A photo of the bridge is presented in Fig. 3a. The mid support is a remnant from the previous bridge and is not utilized. The deck is designed as an unballasted steel grillage consisting of main beams, cross beams and stringers. Wooden sleepers are supported directly by the stringers. A crosssection of the deck is shown in Fig. 3b. The distance between the cross beams is $3.75 \mathrm{~m}$ which is the same for the hangers. The hangers consist of solid steel rods with a diameter of $80 \mathrm{~mm}$. The arch has a circular shape with a radius of $31.9 \mathrm{~m}$ and height of $8.9 \mathrm{~m}$, measured from the top of the main beam to the arch centre line.

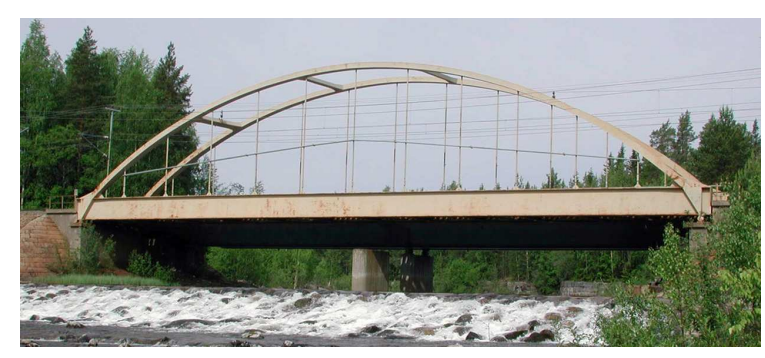

a)

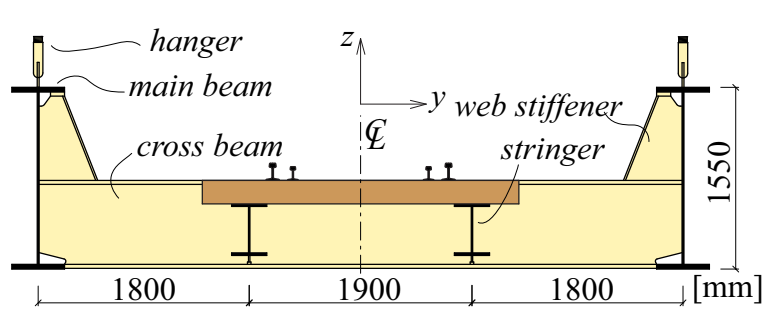

b)

Fig. 3: The Ljungan bridge, a) view, b) cross-section of the steel beam grillage.

During train passage, resonant vibration of several hangers was observed, resulting in a significant decrease in estimated fatigue service life. The most critical section was found to be a threaded turn buckle connection at the lower part of the hanger, depicted in Fig. 4a-c.

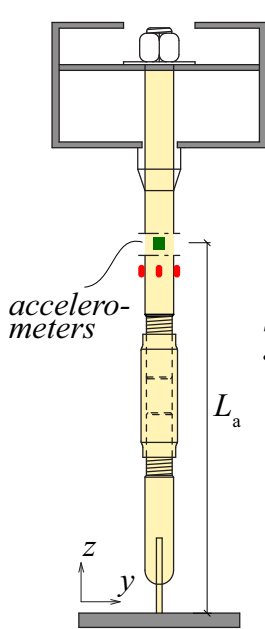

a)

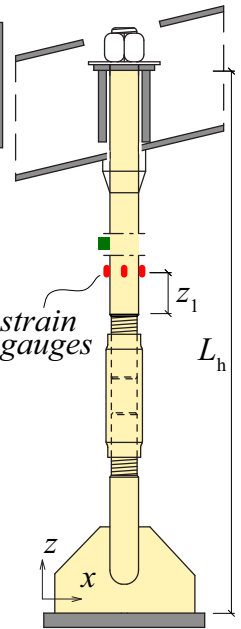

b)

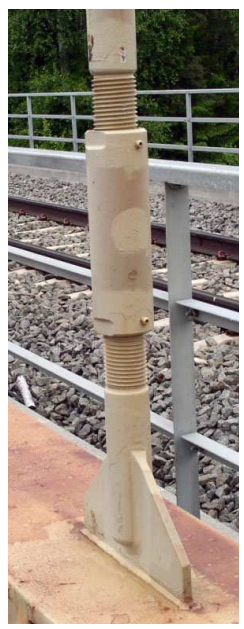

c)

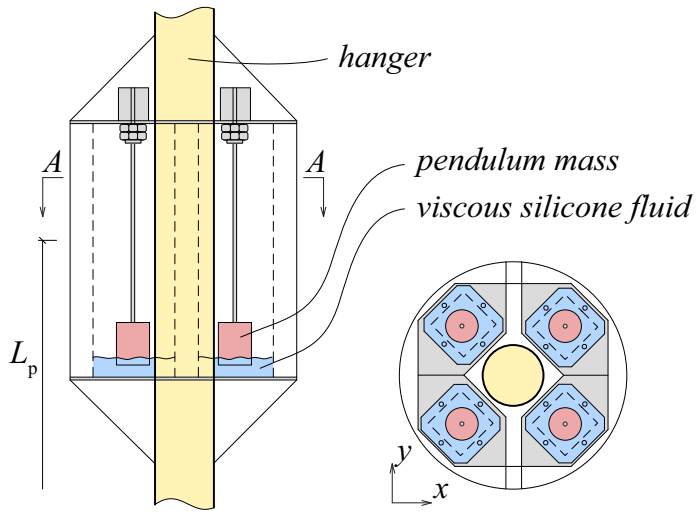

Section A-A e)

Fig. 4: Hanger details, a)-c) instrumentation without damper systems, d-e) current pendulum dampers on hanger 3-9, reproduced from [3]. 
The response was evaluated both by field measurements and numerical simulations. Resonant loading in combination with low damping showed that more than $50 \%$ of the cumulative fatigue damage of the longer hangers were related to free vibrations [1,5]. In 2005, a system of external pendulum dampers was installed on the hangers, illustrated in Fig. 4d-e [2,3]. The pendulum dampers are tuned to the natural frequencies of the unloaded bridge and based on free vibration measurements the average damping ratio was increased from $0.2 \%$ to $3.5 \%$ [3]. Due to the boundary conditions of the hangers, different frequencies in the longitudinal and transverse direction are obtained. The hangers are numbered 1 to 6 with a length from $1.30 \mathrm{~m}$ to $7.15 \mathrm{~m}$.

\subsection{Finite element models}

Although the dynamic behaviour of the bridge is three-dimensional, the essential response can be illustrated using a 2D approach. A 2D FE-model of the bridge is illustrated in Fig. 5, constituting a symmetric half of the bridge. All components are modelled as Euler-Bernoulli beam elements. The hangers are assumed rigidly connected to the main beam but hinged to the arch. This has shown to give a good fit of natural frequencies compared to the field measurements. The dynamic response from passing trains is modelled as vertical point loads applied directly on the stringer beam. The cross beams are modelled as vertical springs with a stiffness calculated on basis of a simply supported beam subjected to two point loads. During train passage, the increase in axial force of the hangers results in a significant change in natural frequency. This is accounted for by a geometrically nonlinear analysis. As a consequence, modal superposition is not applicable and a direct time integration scheme is used. A structural damping of $0.2 \%$ is included and the resulting damping matrix is based on Rayleigh damping.

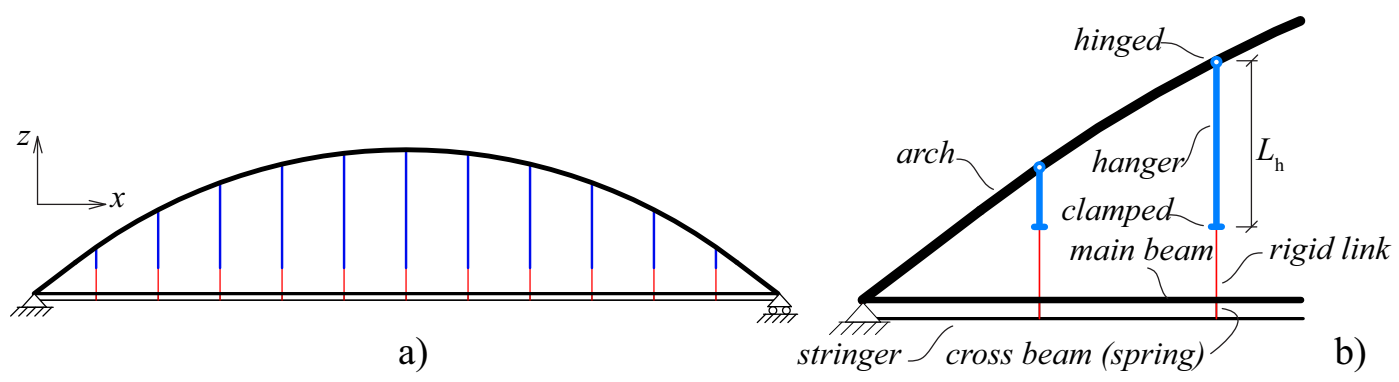

Fig. 5: 2D FE-model of the bridge, a) elevation, $b$ ) details and connections.

External dampers are modelled as illustrated in Fig. 6. The damper mass $m_{\mathrm{d}}$ is modelled as a lumped nodal mass. The TMD-model is connected to the hanger by springs and dashpots in the longitudinal direction and is fully constrained to follow the motion of the hanger in vertical direction. The relative motion between the two nodal masses is also constrained. For the pendulum model, the nodal mass is connected by a beam element with stiffness $E I_{\mathrm{d}}$, which at the upper end is rigidly connected to the hanger, hence acting as a clamped pendulum. An additional viscous damping is included by a horizontal damper $c_{\mathrm{d}}$.
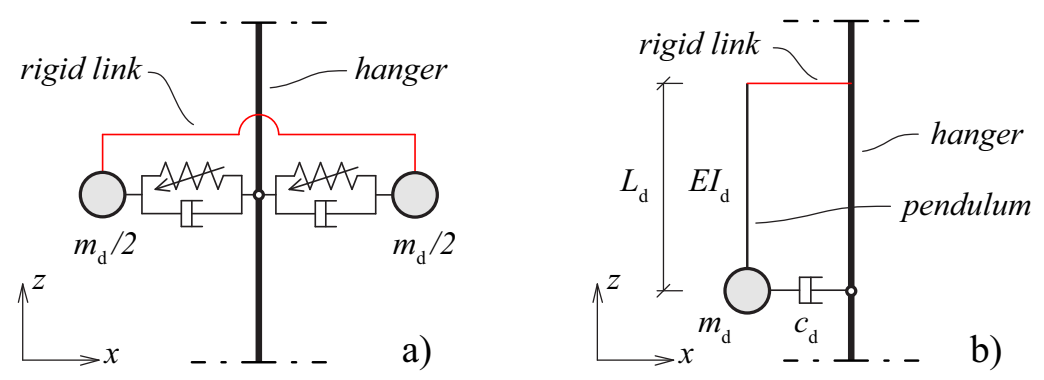

Fig. 6: Detail of dampers in the 2D-model, a) variable stiffness TMD, b) clamped pendulum. 
The properties of the damper models are determined by Eq. (1), Eq. (7) and Eq. (8). When modelling the adaptive TMD, the stiffness matrix is incrementally updated to account for the change in spring stiffness. The analysis is performed using the commercial FE-package SOLVIA03 [8] and an adaptive scheme is automated by routines developed in MATLAB [6].

\section{Simulation results}

In this paper, simulations of the dynamic behaviour of hanger 5 are presented. The length of the hanger is $6.95 \mathrm{~m}$ and from field measurements the first longitudinal natural frequency was estimated at $4.3 \mathrm{~Hz}$ with an appertaining damping of $0.15 \%$.

\subsection{MDOF-models, transient dynamics}

Assuming the hanger to be simply supported, Eq. (3) results in $m_{1}=135 \mathrm{~kg}$ and $k=100 \mathrm{kN} / \mathrm{m}$. Further, Eq. (1) gives $c=11 \mathrm{Ns} / \mathrm{m}$. Due to the low damping, the steady state response is about 300 times the static response. From field measurements, the magnitude of dynamic longitudinal displacement was estimated as $5 \mathrm{~mm}$ based on time integrated accelerations. The duration of the train passage was about $10 \mathrm{~s}$. Using the MDOF-models, a harmonic load block with duration of $10 \mathrm{~s}$ is used in a transient dynamic analysis. The influence of frequency detuning is investigated by a parametric study according to Table 1 , where $f_{1}$ is the natural frequency, $f_{\mathrm{d}}$ the frequency of the damper and $f_{\mathrm{F}}$ the frequency of the load. The magnitude of the load is adjusted to produce a displacement of $5 \mathrm{~mm}$ for the SDOF-model. For both the TMD and pendulum models, a mass $m_{\mathrm{d}}=$ $1 \mathrm{~kg}$ and damping ratio $\zeta_{\mathrm{d}}=1 \%$ is used.

The results in terms of peak displacements are presented in Fig. 7. Due to the low structural damping, the resonance peak of the SDOF-model is rather narrow and a detune of the load frequency results in an off-resonance response and a reduction by a factor of 5 , case b) and d). For a perfectly tuned TMD, the initial response is reduced by a factor of $\sim 10$, case a). However, a detune of either the load frequency or the damper frequency show a significant disimprovement, worse than the detuned SDOF-system. The peak displacement is obtained during the forced vibration and detuning both the damper and the force frequency, case d) results in a force-tuned TMD with similar performance to the perfectly tuned case. However, the following free vibrations will still experience significant oscillation. Comparison of the pendulum dampers show that they both perform similarly to the TMD, except for the detuned pinned pendulum. For the adaptive variable stiffness TMD, frequency estimators based on STFT and STWT are compared. For the perfectly tuned case, all models perform similarly to the passive TMD. In detuning however, the STWT routine performs slightly better and is comparable to the perfectly tuned TMD for all studied cases.

Table 1: Set of parameter studies for frequency variation of the MDOF models.

\begin{tabular}{cccc}
\hline case & $f_{1}(\mathrm{~Hz})$ & $f_{\mathrm{d}}(\mathrm{Hz})$ & $f_{\mathrm{F}}(\mathrm{Hz})$ \\
\hline$a)$ & 4.30 & $f_{1}$ & $f_{1}$ \\
$b)$ & 4.30 & $f_{1}$ & $0.95 f_{1}$ \\
$c)$ & 4.30 & $0.95 f_{1}$ & $f_{1}$ \\
d) & 4.30 & $0.95 f_{1}$ & $0.95 f_{1}$ \\
\hline
\end{tabular}

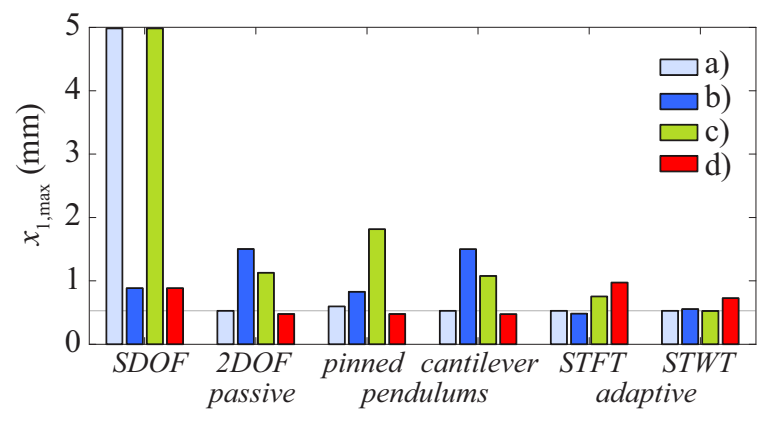

Fig. 7: Peak displacement of the primary structure due to a transient loading block, influence of frequency variation for different TMD models. 


\subsection{D-model, dynamics of passing trains}

The dynamic response of the bridge during train passage is analysed using the 2D FE-model. A freight train consisting of one locomotive and 19 wagons is used. For the locomotive (Swedish Rc4), the axle distance $2.7 \mathrm{~m}$, bogie distance $7.7 \mathrm{~m}$, the total length $15.5 \mathrm{~m}$ and the load $195 \mathrm{kN} / \mathrm{axle}$ are used. Standardized freight train wagons are assumed according to load class D2. This corresponds to an axle distance $1.8 \mathrm{~m}$, bogie distance $9.2 \mathrm{~m}$, length $14.0 \mathrm{~m}$ and an axle load of $225 \mathrm{kN} / \mathrm{axle}$. A similar train passage was recorded during the field measurements, although the exact specifications were not known. The stress components in hanger 5 during the train passage are presented in Fig. 8. The measured response is found to be comparable with the simple 2D-model, although the transverse bending component $\sigma_{\mathrm{Mx}}$ is not accounted for. Comparing the axial stresses indicate that not all wagons of the real train have the same specifications regarding axle load and/or axle distances. Both the measured response and the FE-model demonstrate excessive bending stresses due to resonant loading. It shall be noted that due to the low structural damping, the response may change significantly for a small change in load frequency, e.g. due to different train speed or axle distance. Also, due to different frequencies in the longitudinal and transversal direction, resonance in either direction may be dominant for different speeds.
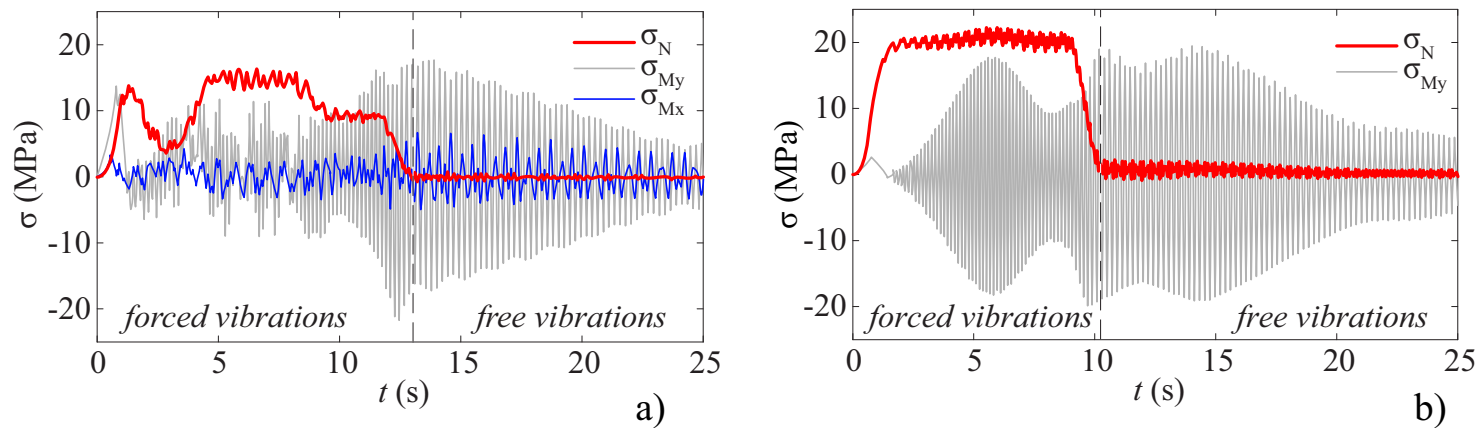

Fig. 8: Stress components in hanger 5 during train passage, a) from field measurements, b) from $2 \mathrm{D} F E$-model, $v=115 \mathrm{~km} / \mathrm{h}$. Results without external dampers.

During train passage, the increased axial force in hanger 5 results in an increase in natural frequency from $4.3 \mathrm{~Hz}$ to about $6.5 \mathrm{~Hz}$. This will cause a passive damper system to be almost completely detuned during the train passage, if tuned based on the unloaded hanger frequency. A comparison of different damper systems is presented in Fig. 9, all based on the 2D FE-model. For clarity, envelopes of the bending stresses are shown. The light shaded area represents the undamped response, as in Fig. 8b.

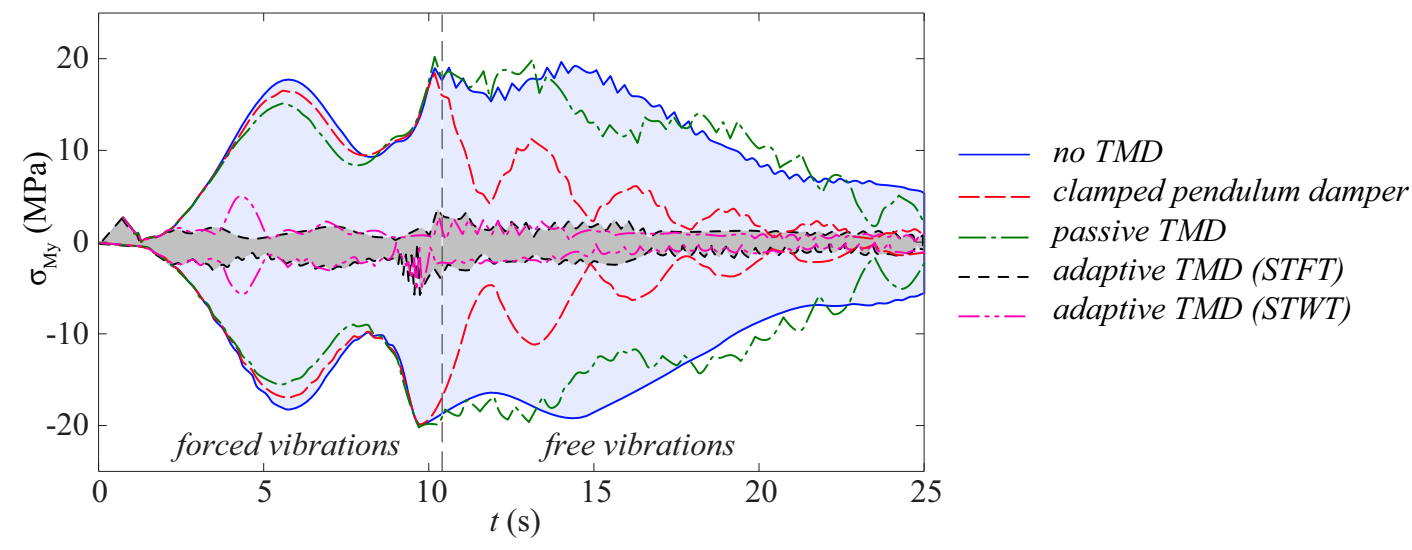

Fig. 9: Bending stress in hanger 5, results from the 2D-model with different damping systems. 
Both the pendulum damper and the passive TMD show little improvement during the forced vibrations. During free vibrations, the pendulum damper performed significantly better than the passive TMD. The darker shaded area represents the working range of the adaptive TMD, proving an overall significant vibration mitigation for both forced and free vibrations.

\section{Conclusions}

In this paper, the use of passive and adaptive damping systems for mitigation of railway bridge dynamics is studied, by means of numerical simulations. Based on MDOF-models, it is shown that a passive TMD is sensitive to detuning, which can be greatly improved by an adaptive variable stiffness TMD. The application of the damper systems is further illustrated for a case study bridge, proven to be susceptible to resonant loading. During train passage, the frequency of vertical hangers change due to increased axial force. Numerical simulations show that passive systems tuned to the frequency of the unloaded bridge will have little influence on the forced vibration but significant influence on the free vibrations. For such configurations, the initial free vibrations have rather high amplitude, affecting the possible vibration mitigation. Using an adaptive system however, the forced vibration is attenuated significantly, causing initially lower amplitudes during free vibration and hence lower amplitudes during both forced and free vibration.

\section{Acknowledgements}

The field measurements in 2003 were commissioned by the Swedish Transport Administration (former Banverket) and performed by KTH. Additional analysis and the development of routines for adaptive vibration damping were performed within the Long Life Bridges project, a Marie Curie Industry-Academia Partnerships and Pathways project funded by the European Commission $7^{\text {th }}$ Framework Programme (IAPP-GA-2011-286276). All funding and support mentioned above is greatly acknowledged.

\section{References}

[1] ANDERSSON, A., MALM R., "Measurement Evaluation and FEM Simulation of Bridge Dynamics", MSc Thesis, KTH Royal Institute of Technology, Sweden, 2004.

[2] HORTMANNS, M., SCHÄFER, N., "Ljungan Bridge, Ånge Sweden - Full Scale Measurements", Technical report (unpublished), Prof. Sedlacek \& Partner, 2005.

[3] HORTMANNS, M., "Ljungan Bridge, Ånge Sweden - Design of dampers for the hangers 3 to 6", Technical report (unpublished), Prof. Sedlacek \& Partner, 2005.

[4] LIEDES, T., "Improving the performance of the semi-active tuned mass damper", PhD-Thesis, University of Oulu, Finland, 2009.

[5] MALM, R., ANDERSSON, A., "Field testing and simulation of dynamic properties of a tied arch railway bridge", Engineering Structures 28(1), 2006.

[6] MATLAB, "MATLAB 2009b Reference manual”, The MathWorks Inc, 2009.

[7] ORMONDROYD, J., DEN HARTOG, J.P., "The theory of the dynamic vibration absorber", ASME, Journal of Applied Mechanics 50, 1928.

[8] SOLVIA, "SOLVIA-PRE for Stress Analysis, User Manual”, Report SE 03-1, SOLVIA Finite Element Systems Version 03, 2008.

[9] TSAI, H.-C., LIN, G.-C., "Explicit formulae for optimum absorber parameters for forceexcited and viscously damped systems”, Journal of Sound and Vibration 176(5), 1994. 